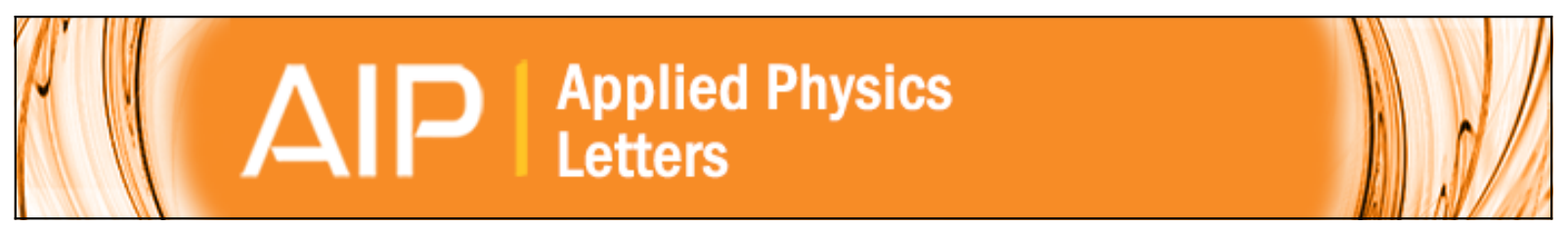

\title{
Breaking the configurational anisotropy in Fe single crystal nanomagnets
}

A. Gomez, F. Cebollada, F. J. Palomares, N. Sanchez, E. M. Gonzalez, J. M. Gonzalez, and J. L. Vicent

Citation: Applied Physics Letters 104, 102406 (2014); doi: 10.1063/1.4868401

View online: http://dx.doi.org/10.1063/1.4868401

View Table of Contents: http://scitation.aip.org/content/aip/journal/apl/104/10?ver=pdfcov

Published by the AIP Publishing

\section{Articles you may be interested in}

Mechanism of reversing the Neel domain walls in the Co nanostripes with transverse magnetic anisotropy

Appl. Phys. Lett. 101, 252412 (2012); 10.1063/1.4772981

Switching behavior of lithographically fabricated nanomagnets for logic applications

J. Appl. Phys. 111, 07B911 (2012); 10.1063/1.3676220

Manipulation of magnetization reversal of Ni81Fe19 nanoellipse arrays by tuning the shape anisotropy and the magnetostatic interactions

J. Appl. Phys. 111, $07 B 909$ (2012); 10.1063/1.3676215

Transition of magnetocrystalline anisotropy and domain structure in epitaxial $\mathrm{Fe}(001)$ nanomagnets

J. Appl. Phys. 93, 7432 (2003); 10.1063/1.1558256

Coherent magnetization reversal of nanoparticles with crystal and shape anisotropy

J. Appl. Phys. 89, 507 (2001); 10.1063/1.1323519

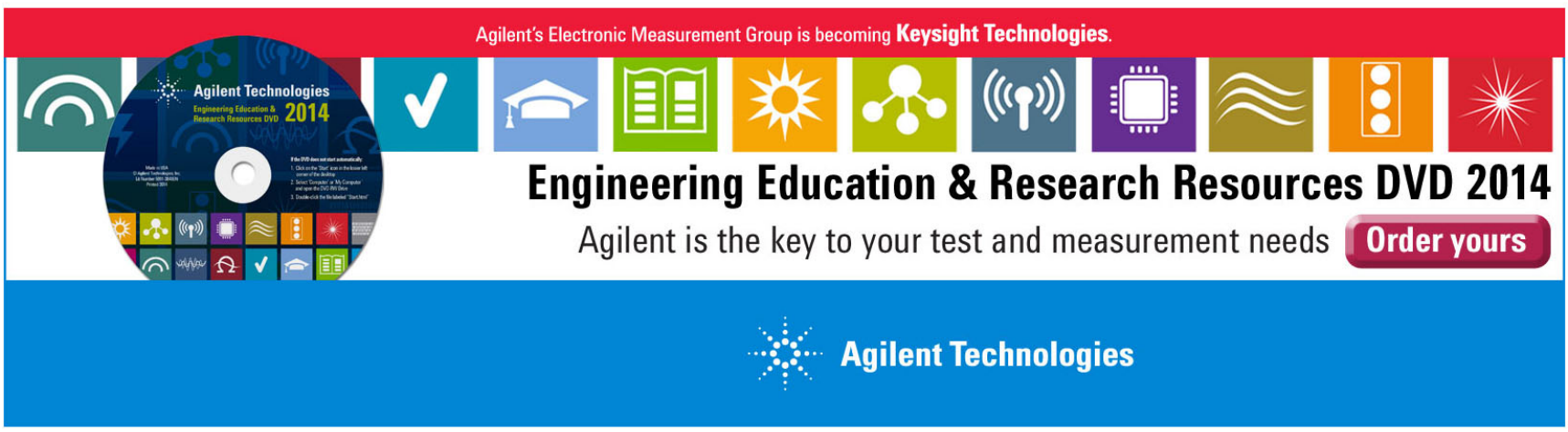




\title{
Breaking the configurational anisotropy in Fe single crystal nanomagnets
}

\author{
A. Gomez, ${ }^{1}$ F. Cebollada, ${ }^{2}$ F. J. Palomares,${ }^{3}$ N. Sanchez, ${ }^{3}$ E. M. Gonzalez, ${ }^{1,4}$ \\ J. M. Gonzalez, ${ }^{3}$ and J. L. Vicent ${ }^{1,4}$ \\ ${ }^{1}$ Departamento de Física de Materiales, Facultad CC. Físicas, Universidad Complutense, 28040 Madrid, Spain \\ ${ }^{2}$ POEMMA-CEMDATIC, ETSI de Telecomunicación, Universidad Politécnica de Madrid, 28040 Madrid, \\ Spain \\ ${ }^{3}$ Instituto de Ciencia de Materiales de Madrid (CSIC), Sor Juana Inés de la Cruz, 3, 28049 Madrid, Spain \\ ${ }^{4}$ IMDEA-Nanociencia, Cantoblanco, 28049 Madrid, Spain
}

(Received 14 January 2014; accepted 28 February 2014; published online 12 March 2014)

\begin{abstract}
In this work, we improve the ability to tailor the switching mechanism in nanomagnets by introducing an additional, highly controlled source of anisotropy: magnetocrystalline anisotropy. We analyze the vortex dynamics in single crystal Fe nanotriangles with different orientations of the crystalline axes. By experimental studies and simulation, we show that the angular dependence of the vortex annihilation field springs from the convolution of the crystalline and configurational anisotropies. In contrast, the remanence and the nucleation field present a much simpler behavior controlled by the existence of a single symmetry axis when shape and crystalline orientation are taken into account. (C) 2014 AIP Publishing LLC. [http://dx.doi.org/10.1063/1.4868401]
\end{abstract}

Magnetic nanostructures have become a highly active research area due to their unique physical behavior and to their relevance in established and emerging technological fields such as spintronics, magnonics, magnetic storage, nanobiomagnetism, and high frequency and superconducting devices. ${ }^{1-7}$ A key issue in tailoring the hysteresis processes of magnetic nanostructures lies in the control of the different anisotropy energy contributions and their corresponding effective fields. ${ }^{89}$ One of the main energy contributions in magnetic nanodots is due to the internal dipolar (demagnetizing) fields. These give rise to the so-called configurational anisotropy, ${ }^{10-12}$ which is related to the geometry of the dots. The reduction of the large dipolar fields for dots in the range between the single and multidomain regime is accomplished through the formation closed-flux structures called vortices. ${ }^{13-17}$ The hysteresis of vortex dynamics is characterized by two large magnetization jumps associated with the nucleation and annihilation of the vortices, respectively, and very low remanence due to their closed flux structure. ${ }^{10}$ Vortices nucleate at the dot edges upon decreasing the applied field from saturation at a field value called nucleation field $\mathrm{H}_{n}$. They traverse the dot when the applied field is reversed and they are finally expelled when the annihilation field $\mathrm{H}_{\mathrm{ann}}$ is reached. ${ }^{18-20}$ Vortex dynamics can be controlled using different strategies. As an example, the nucleation and annihilation sites can be selected and their corresponding nucleation and annihilation fields can be tuned by choosing the geometry of the dots in combination with the orientation of the applied field. ${ }^{19-25}$ Most studies have emphasized the relevance of the dipolar fields in controlling the hysteresis of dots. So far we know, the role of the magnetocrystalline anisotropy energy (MAE) has not been usually considered since most of the experimental studies in the literature deal with dots patterned on Permalloy ${ }^{14,15,18,23}$ (low MAE) or on isotropic polycrystalline films ${ }^{16,22,25}$ (no preferential crystalline orientation). However, MAE might become a major factor in the magnetization mechanisms of single crystal dots and, in fact, theoretical calculations indicate that it strongly affects the hysteresis parameters. ${ }^{26}$
In this paper, we present an experimental study and simulation of the interplay between the MAE and magnetostatic energy (MSE) contributions to the hysteresis processes in two arrays of single crystal Fe nanotriangles with different orientations of the crystalline axes. We will show that the annihilation field presents a complex behavior resulting from the combined symmetries of the MAE (four-fold) and of the configurational anisotropy (six-fold). In contrast, the angular dependence of the remanence and nucleation field exhibits a two-fold structure around the single symmetry axis present in each triangle when shape and crystalline orientation are taken into account.

Two arrays of submicron dots were patterned by electron beam lithography on a $\mathrm{Au}(001) / \mathrm{Fe}(001) / \mathrm{MgO}(001)$ thin film grown by pulsed laser deposition (Fe) and molecular beam epitaxy (Au). Previous studies proved the single crystal nature of the $\mathrm{Fe}$ and Au layers, $25 \mathrm{~nm}$ and $10 \mathrm{~nm}$ thick, respectively. ${ }^{9,27}$ Each array consists of a square lattice $(1.4 \mu \mathrm{m}$ lattice parameter) of equilateral triangles of $600 \mathrm{~nm}$ side. The large interdot separation, over twice their size, allows us to neglect the dipolar interactions between neighboring triangles. $^{26,28}$ The $\mathrm{Fe}$ easy magnetocrystalline axes [100] and [010] were either parallel and perpendicular (array A) or at $45^{\circ}$ (array B) with respect to the base of the triangles (Fig. 1). As a consequence of the combined six-fold (geometry) and four-fold (crystalline) symmetries, the triangles in both arrays have a single symmetry axis corresponding to the base-to-tip line. A magneto-optic Kerr effect setup was used to measure the in-plane angular dependence of the hysteresis properties of the arrays, under a maximum applied field of 5 $\mathrm{kOe}$ at room temperature. Micromagnetic simulations were carried out using OOMMF code $\mathrm{e}^{29}$ and typical values of the Fe parameters: saturation magnetization $\mathrm{M}_{\mathrm{S}}=1.7 \times 10^{6}$ $\mathrm{A} / \mathrm{m}$, exchange constant $\mathrm{A}=2.1 \times 10^{-11} \mathrm{~J} / \mathrm{m}$, and anisotropy constant $\mathrm{K}=4.8 \times 10^{4} \mathrm{~J} / \mathrm{m}^{3}$. ${ }^{30}$

The hysteresis loops of both arrays, measured with the applied field along the base $\left[\theta=0^{\circ}\right.$, see sketch in Fig. 1(a)] and base-to-tip $\left(\theta=90^{\circ}\right)$ directions, are shown in Fig. 1. As 
can be seen, all of them are constricted, with two large magnetization jumps. Upon decreasing the applied field from saturation, the first jump takes the magnetization almost from (positive) saturation to a value around zero whereas the second one approaches it to (negative) saturation. The first jump corresponds to the nucleation of a closed flux, vortex structure which appears in order to reduce the large MSE required to keep the nanotriangles uniformly magnetized..$^{10,13,15}$ The nucleation field $\mathrm{H}_{\mathrm{n}}$ associated with the first jump was evaluated from the magnetization slope of the upper branch of the loops, see e.g., Fig. 1(c). It is close to zero in the loops of array $\mathrm{A}$ and in the $\theta=0^{\circ}$ loop of array $\mathrm{B}$; in contrast, the vortex nucleation in the $\theta=90^{\circ}$ loop of array B occurs at a positive field $\mathrm{H}_{\mathrm{n}} \approx 120$ Oe. As shown in previous studies, ${ }^{31,32}$ carried out in low anisotropy materials, the directions parallel to the triangle sides correspond to easy axes from the point of view of the MSE. On the contrary, the MSE is maximal when the triangle is magnetized along the base-to-tip or shape-equivalent directions due to the large density of poles at the triangle base and to the large dipolar (demagnetizing) field they create. This induces the well-known six-fold configurational anisotropy of nanotriangles, with an energy

(a)

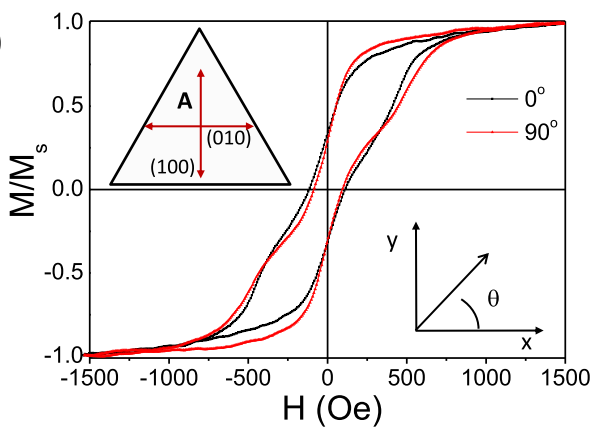

(b)

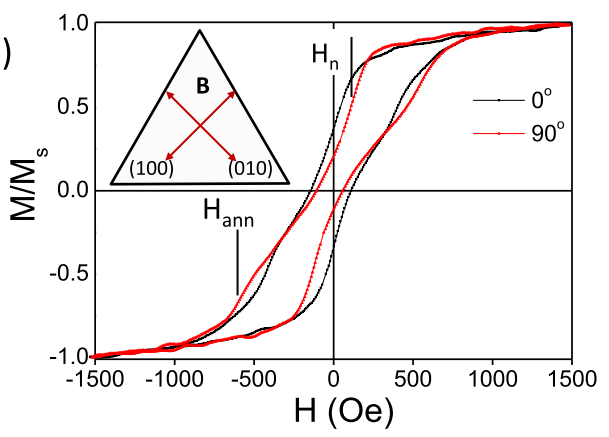

(c)

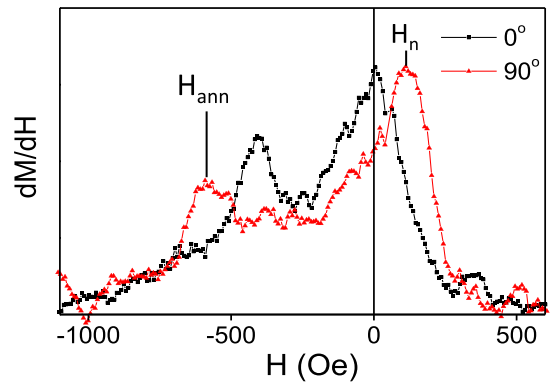

FIG. 1. Hysteresis loops with the applied field along the base $\left(\theta=0^{\circ}\right)$ and along the base-to-tip direction $\left(\theta=90^{\circ}\right)$ for arrays A (a) and B (b). The orientation of the crystalline axes is shown for each array and inset in (a) shows the angle scheme employed for both arrays. (c) Field derivative of the demagnetization branch of the hysteresis loops of array $\mathrm{B}$. The nucleation field $\mathrm{H}_{\mathrm{n}}$ and annihilation field $\mathrm{H}_{\mathrm{ann}}$ of array $\mathrm{B}\left(\theta=90^{\circ}\right)$ are labeled in (b) and (c). maximum every $60^{\circ} .{ }^{32}$ In the case of array $\mathrm{B}$, the magnetocrystalline anisotropy energy (MAE) is also maximum for $\theta=90^{\circ}$, i.e., this direction corresponds to a hard MSE and MAE axis simultaneously, and, consequently, vortices nucleate even though the applied field is positive. The equivalent direction for array A is, on the other hand, an easy axis from the point of view of the MAE. Both the applied field and the magnetocrystalline anisotropy help to keep the magnetization vertical and vortices nucleate only when the applied field is reduced to a lower value, close to zero in our case. Fig. 2(a) shows the angular dependence of the nucleation field, which has a constant value $\mathrm{H}_{\mathrm{n}} \approx 120 \mathrm{Oe}$ in an angular region approximately $\pm 20^{\circ}$ around $\theta=90^{\circ}$ in the case of array $\mathrm{B}$ and drops to zero out of it. The nucleation field of array $A$ is essentially null $\left(-15 \mathrm{Oe}<\mathrm{H}_{\mathrm{n}}<10 \mathrm{Oe}\right)$ at all angles, which is due to the fact that there are no directions corresponding to a coincident MAE and MSE maximum.

The vortex nucleation at positive fields in array $\mathrm{B}$ around $\theta=90^{\circ}$ leads to a steep magnetization decrease and thus to the minimum remanence-to-saturation ratio, $\mathrm{M}_{\mathrm{R}} / \mathrm{M}_{\mathrm{S}} \approx 0.15$, as can be seen in Fig. 2(b). This low remanence value is linked to an almost fully closed flux structure occurring after the nucleation process is completed. On the contrary, when the nucleation field is at its peak at $\mathrm{H}=0$, the magnetization structures are not strictly closed and thus a higher remanence was obtained. This is consistent with the higher remanence-to-saturation ratio of array B in the angular region far from $\theta=90^{\circ}$ and also in the whole angular range for array A, as shown in Fig. 2(b). In both cases, the nucleation field is close to zero and $\mathrm{M}_{\mathrm{R}} / \mathrm{M}_{\mathrm{S}}$ varies between 0.32 and 0.44 .

To get a further insight into the remanent magnetization configuration, we measured the transverse component of the remanence, i.e., the $\mathrm{M}_{\mathrm{RY}}$ component after saturating along $\mathrm{x}$ (see axes on Fig. 1) and vice versa, for both arrays. The remanent state was induced in each array by applying a $5 \mathrm{kOe}$ saturating magnetic field and then switching it off. The array was then rotated $90^{\circ}$ and a new hysteresis loop was
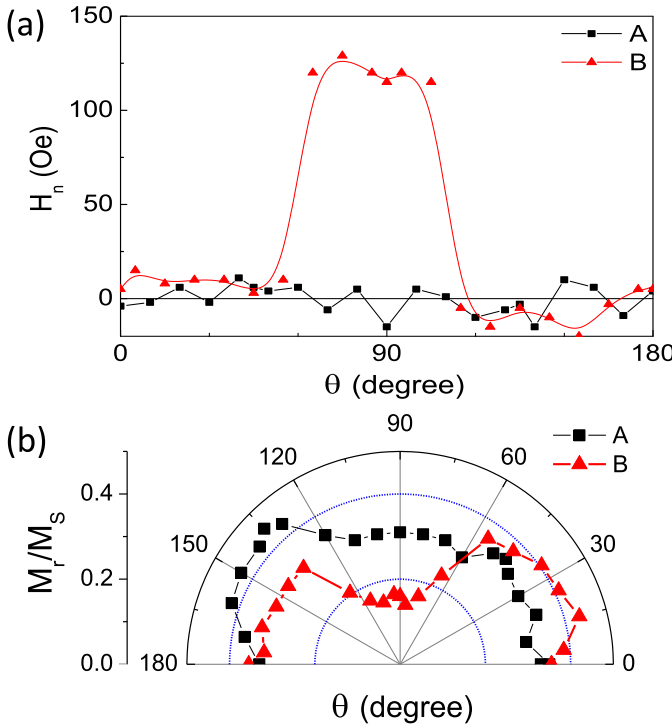

FIG. 2. Angular dependence of the (a) nucleation field, Hn, and (b) remanence, $\mathrm{M}_{\mathrm{r}} / \mathrm{M}_{\mathrm{s}}$, for both arrays. Black squares correspond to array $\mathrm{A}$ and red triangles to array B in both graphs. 


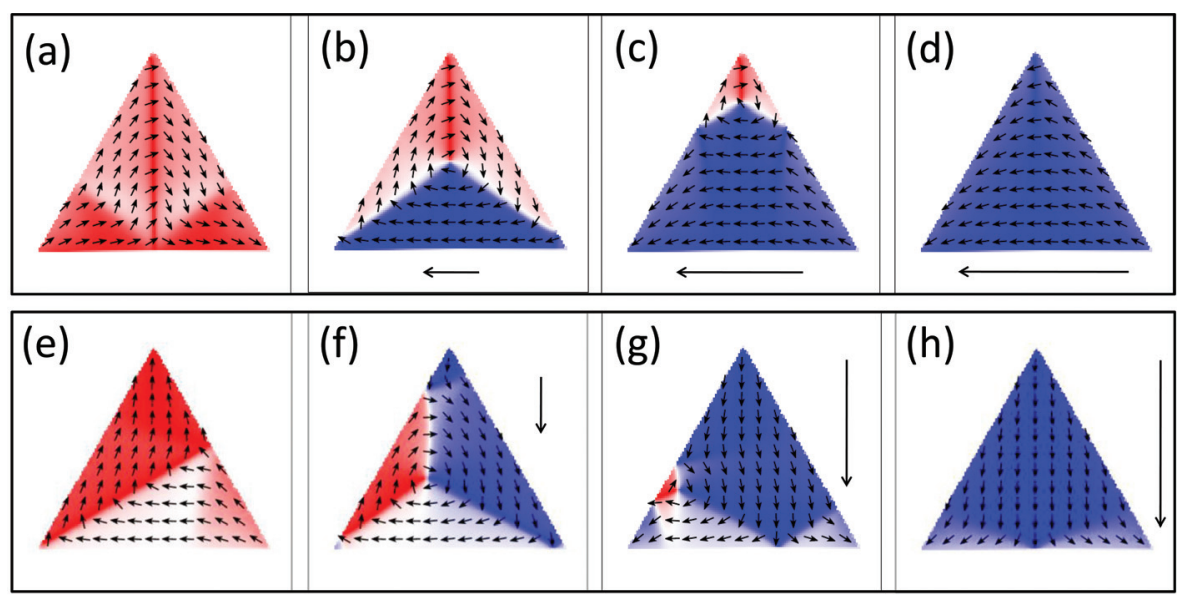

FIG. 3. Magnetization configurations simulated for array A, with the applied field either along the base $(\mathrm{a}-\mathrm{d})$ or along the base-to-tip direction (e-h). Different states are shown from remanence after positive saturation (left) to negative saturation (right); arrows indicate the direction and amplitude of the applied magnetic field.

measured. The starting point of the new hysteresis loop corresponds to the transverse component of the remanent magnetization after the initial saturation. The transverse component of the magnetization was null in all four cases. According to the micromagnetic simulations, after positive saturation along the base $\left(\theta=0^{\circ}\right)$ a remanence configuration resembling a "C" state is obtained, such as that shown in Fig. 3(a), corresponding to array A. In this state, the transverse (vertical) magnetization component is null, in agreement with the experiment. A vortex nucleates at the center of the base and, upon the application of a negative field, it moves up to the center of the triangle, Fig. 3(b), it is annihilated at the tip, Fig. 3(c) and, finally, the triangle is saturated, Fig. 3(d). When the triangles are saturated from base to tip $\left(\theta=90^{\circ}\right)$, the resulting remanent configuration is similar to a "buckle" state 31,32 (the magnetization bends parallel to the base and then rotates to the tip), as shown in Fig. 3(e). No symmetric " $y$ " state is stable according to simulations, in agreement with previous results on permalloy which indicate that this state can only be stabilized under very restrictive conditions (triangle size below $200 \mathrm{~nm}$ and sharp corners). ${ }^{31,32}$ The vortices nucleate at one of the sides of the triangles and they move to the center, Fig. 3(f), and exit at the opposite corner, Fig. 3(g), before saturation, Fig. 3(h). As shown in Fig. 3(e), the buckle state is not left-right symmetric and, consequently, the transverse (horizontal) magnetization component is not null. However, there is no preference for the right or left side as nucleation site in each triangle, which means that, after vertical saturation, both left and right nucleation sides are equally probable. This accounts for the null transverse component of the magnetization in both arrays even when they are vertically saturated.

The annihilation field $\mathrm{H}_{\mathrm{ann}}$ is the field required to break the vortex structure and to align the magnetization with the field. To account for the angular variation of the annihilation field, we have to make several considerations. After vortex annihilation, the magnetization is close to uniform and the MSE increases due to the poles appearing at the edges. This increase is maximum (minimum) along the base-to-tip (base) and equivalent directions. ${ }^{32}$ The uniform magnetization leads to a decrease in the exchange energy. Finally, we have to consider that keeping the magnetization parallel to a hard MAE axis requires a higher field than along an easy axis. The annihilation field of array A [Fig. 4(a)] varies from
390 Oe to 550 Oe, approximately, and its maxima appear at the hard MSE directions, $\theta=30^{\circ}, \theta=90^{\circ}$, and $\theta=150^{\circ}$. The fact that $\theta=90^{\circ}$ corresponds to a relative maximum in spite of the magnetization lying along an easy MAE axis makes clear the higher weight of the MSE in the magnetization mechanism. The absolute maxima at $\theta=30^{\circ}$ and $\theta=150^{\circ}$ are related to the proximity of the hard MSE and MAE directions, which are just $15^{\circ}$ apart. The annihilation field of array B [Fig. 4(b)] varies between $400 \mathrm{Oe}$ and $580 \mathrm{Oe}$, approximately, with an absolute maximum at $\theta=90^{\circ}$-coincident with a hard MSE and MAE axis- and an absolute minimum

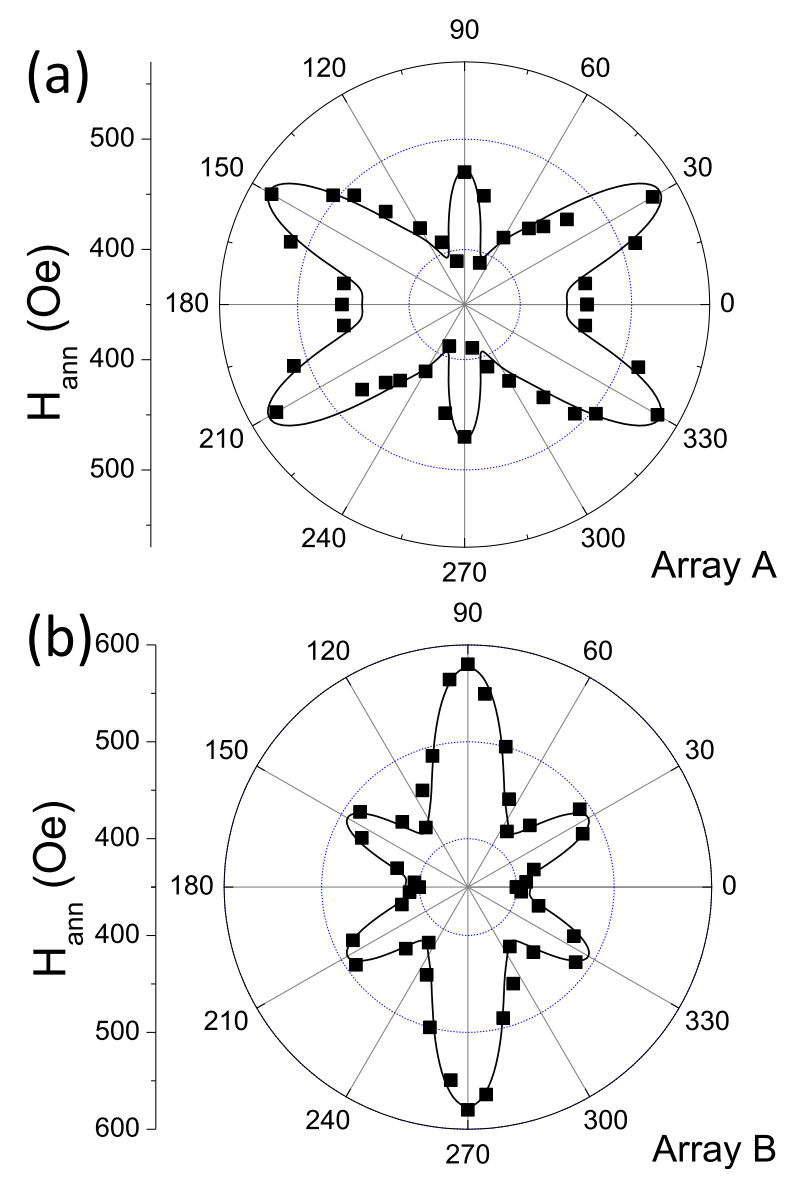

FIG. 4. Angular dependence of the annihilation field $\left(\mathrm{H}_{\mathrm{ann}}\right)$ of array A (a) and array B (b). Dots correspond to experiment and line shows the theoretical fit. Note: $180^{\circ}-360^{\circ}$ interval is duplicated from the $0^{\circ}-180^{\circ}$ interval for clarity. 
when the field is applied along the base of the triangles, which is an easy MSE but hard MAE direction, which confirms the predominance of the MSE. Two relative maxima appear very close to $\theta=30^{\circ}$ and $\theta=150^{\circ}$, i.e., almost coincident with the two remaining hard MSE axes.

To analyze the angular dependence of the annihilation field, we have taken into account the functional form of the in-plane MAE energy (four-fold), of the configurational anisotropy (six-fold) and second order harmonics of both. We have also taken into account that the annihilation field has in itself inversion symmetry (two-fold). The $\mathrm{Fe}(001)$ in-plane MAE, for array A, can be written as ${ }^{33}$

$$
E_{a}=\frac{K_{1}}{8}(1-\cos 4 \theta)
$$

where $\mathrm{K}_{1}$ is the first order anisotropy constant of Fe. From this expression, the in-plane anisotropy, considered as the energy difference between the hard and easy in-plane directions, is $\Delta \mathrm{E}_{\mathrm{a}}=\mathrm{K}_{1} / 4$. The MSE is proportional to the square saturation magnetization $\mathrm{M}_{\mathrm{S}}$

$$
E_{m}=\frac{1}{2} \mu_{0} M_{S}^{2} \gamma(1-\cos 6 \theta),
$$

with the factor $\gamma$ giving the energy difference in MSE between a hard and an easy direction

$$
\Delta E_{m}=\mu_{0} M_{S}^{2} \gamma
$$

The annihilation field of array A was then fitted to the following expression:

$$
\begin{aligned}
H_{A N N}= & D+\alpha\left[A_{1}(1-\cos 4 \theta)+A_{2}(1-\cos 8 \theta)\right. \\
& +B_{1}(1-\cos 6 \theta)+B_{2}(1-\cos 12 \theta) \\
& -C(1-\cos 2 \theta)] .
\end{aligned}
$$

In this expression $\mathrm{D}$ and $\alpha$ are constants, $\mathrm{A}_{1}$ is proportional to $\mathrm{K}_{1} / 8, \mathrm{~B}_{1}$ to $\gamma \mu_{0} \mathrm{M}_{\mathrm{S}}^{2} / 2, \mathrm{C}$ is the coefficient that accounts for the field two-fold symmetry, and $\mathrm{A}_{2}$ and $\mathrm{B}_{2}$ are the coefficients associated with the second harmonics of the MAE and MSE, respectively. In the case of array B, a similar expression was used with the MAE terms rotated $45^{\circ}$. The fitting of the experimental data (Fig. 4) yielded essentially a single set of parameters valid for both arrays: for array $\mathrm{A}(\mathrm{B}$, when different), $\mathrm{D}=370 \quad$ (375) Oe, $\alpha=30 \quad$ (28) Oe, $\mathrm{A}_{1}=0.7$, $\mathrm{B}_{1}=1.7, \mathrm{~A}_{2}=-0.1, \mathrm{~B}_{2}=-0.45(-0.4)$, and $\mathrm{C}=1.2$. We can compare the MSE and the in-plane MAE, with a clear predominance of the first over the second, $\Delta \mathrm{E}_{\mathrm{m}} / \Delta \mathrm{E}_{\mathrm{a}} \approx 2.4$. Using the $\mathrm{M}_{\mathrm{S}}$ and $\mathrm{K}_{1}$ of $\mathrm{Fe}$, the configurational anisotropy coefficient of expression (2) results $\frac{1}{2} \mu_{0} M_{S}^{2} \gamma \approx 1.45 \times 10^{4} \mathrm{~J} / \mathrm{m}^{3}$ and a value of $\gamma \approx 7.8 \times 10^{-3}$ was obtained. These calculations confirm the predominance of the MSE over the MAE, not only in low anisotropy dots or polycrystalline Co $\operatorname{dots}^{16,22,25}$ but also in dots fabricated on single crystal films. ${ }^{27}$

In summary, we have found the way to modify the sixfold symmetry of the configurational anisotropy by choosing the appropriate direction of the magnetocrystalline axes. We have analyzed the relative weight of the MAE and of the
MSE in the magnetization processes of two arrays of single crystal triangular nanodots with different orientations of the crystalline axes. We have shown that the nucleation field and the remanence of array A, in which the hard MAE and MSE axes do no coincide, show a weak angular dependence, the former being close to cero. In contrast, the nucleation field and remanence of array B present a uniaxial symmetry with maximum nucleation field and minimum remanence in a narrow angular region around the single hard MSE and MAE axis. Out of this region the nucleation field drops to zero, as in array $\mathrm{A}$, and the remanence rises to values which are also close to those of array A. The annihilation field exhibits a complex angular dependence, clearly dominated by the sixfold symmetry of the configurational anisotropy and modulated by the MAE. In both arrays, the maximum annihilation field is always obtained along or very close to the hard MSE directions. The analysis of the angular evolution of the annihilation field allowed us to estimate the value of the configurational anisotropy constant, which is over twice the value of the Fe in-plane magnetocrystalline anisotropy.

We thank the financial support by Spanish MINECO, Grant Nos. MAT2010-18432, MAT2010-21088-C03-03, FIS2008-06249 (Grupo consolidado), CONSOLIDER CSD2007-00010, FUNCOAT CSD-2008-0023, and CAM Grant No. S2009/MAT1726.

${ }^{1}$ J. I. Martin, J. Nogues, K. Liu, J. L. Vicent, and I. K. Schuller, J. Magn. Magn. Mater. 256, 449 (2003).

${ }^{2}$ S. D. Bader, Rev. Mod. Phys. 78, 1 (2006).

${ }^{3}$ C. H. Sim, M. Moneck, T. Liew, and J.-G. Zhu, J. Appl. Phys. 111, 07C914 (2012).

${ }^{4}$ J. W. Lau and J. M. Shaw, J. Phys. D: Appl. Phys. 44, 303001 (2011).

${ }^{5}$ P. S. Keatley, V. V. Kruglyak, P. Gangmei, and R. J. Hicken, Philos. Trans. R. Soc., A 369, 3115 (2011).

${ }^{6}$ B. Lenk, H. Ulrichs, F. Garbs, and M. Münzenberg, Phys. Rep. 507, 107 (2011).

${ }^{7}$ A. Gomez, E. M. Gonzalez, M. Iglesias, N. Sanchez, F. J. Palomares, F. Cebollada, J. M. Gonzalez, and J. L. Vicent, J. Phys. D: Appl. Phys. 46, 095302 (2013).

${ }^{8}$ T. Thomson, G. Hu, and B. D. Terris, Phys. Rev. Lett. 96, 257204 (2006).

${ }^{9}$ E. Paz, F. Cebollada, F. J. Palomares, F. Garcia-Sanchez, and J. M. Gonzalez, Nanotechnology 21, 255301 (2010).

${ }^{10}$ R. P. Cowburn, J. Phys. D 33, R1 (2000).

${ }^{11}$ R. P. Cowburn, A. O. Adeye, and H. E. Welland, Phys. Rev. Lett. 81, 5414 (1998)

${ }^{12}$ M. E. Schabes and H. N. Bertram, J. Appl. Phys. 64, 1347 (1988).

${ }^{13}$ T. Shinjo, T. Okuno, R. Hassdorf, K. Shigeto, and T. Ono, Science 289, 930 (2000).

${ }^{14}$ A. Barman, S. Barman, T. Kimura, Y. Fukuma, and Y. Otani, J. Phys. D: Appl. Phys. 43, 422001 (2010).

${ }^{15}$ R. P. Cowburn, D. K. Koltsov, A. O. Adeye, H. E. Welland, and D. M. Tricker, Phys. Rev. Lett. 83, 1042 (1999).

${ }^{16}$ I. L. Prejbeanu, M. Natali, L. D. Buda, U. Ebels, A. Lebib, Y. Chen, and K. Ounadjela, J. Appl. Phys. 91, 7343 (2002).

${ }^{17}$ I. V. Roshchin, C.-P. Li, H. Suhl, X. Batlle, S. Roy, S. K. Sinha, S. Park, R. Pynn, M. R. Fitzsimmons, J. Mejia-Lopez, D. Altbir, A. H. Romero, and I. K. Schuller, Europhys. Lett. 86, 67008 (2009).

${ }^{18}$ M. Schneider, H. Hoffmann, and J. Zweck, Appl. Phys. Lett. 79, 3113 (2001).

${ }^{19}$ N. M. Vargas, S. Allende, B. Leighton, J. Escrig, J. Mejia-Lopez, D. Altbir, and I. K. Schuller, J. Appl. Phys. 109, 073907 (2011).

${ }^{20}$ M. Jaafar, R. Yanes, D. Perez de Lara, O. Chubykalo-Fesenko, A. Asenjo, E. M. Gonzalez, J. V. Anguita, M. Vazquez, and J. L. Vicent, Phys. Rev. B 81, 054439 (2010).

${ }^{21}$ N. B. Leighton, N. M. Vargas, D. Altbir, and J. Escrig, J. Magn. Magn. Mater. 323, 1563 (2011). 
${ }^{22}$ R. K. Dumas, T. Gredig, C. P. Li, I. K. Schuller, and K. Liu, Phys. Rev. B 80, 014416 (2009).

${ }^{23}$ K. M. Wu, L. Horng, J. F. Wang, J. C. Wu, Y. H. Wu, and C. M. Lee, Appl. Phys. Lett. 92, 262507 (2008).

${ }^{24}$ M. Jaafar, R. Yanes, A. Asenjo, O. Chubykalo-Fesenko, M. Vazquez, E. M. Gonzalez, and J. L. Vicent, Nanotechnology 19, 285717 (2008).

${ }^{25}$ R. K. Dumas, D. A. Gilbert, N. Eibagi, and K. Liu, Phys. Rev. B 83, 060415(R) (2011).

${ }^{26}$ J. Mejia-Lopez, D. Altbir, A. H. Romero, X. Battle, I. V. Roshchin, C.-P. Li, and I. K. Schuller, J. Appl. Phys. 100, 104319 (2006).

${ }^{27}$ E. Paz, F. Cebollada, F. J. Palomares, J. M. Gonzalez, J. S. Martins, N. M. Santos, and N. A. Sobolev, J. Appl. Phys. 111, 123917 (2012).
${ }^{28}$ V. Novosad, M. Grimsditch, K. Yu. Guslienko, P. Vavassori, Y. Otani, and S. D. Bader, Phys. Rev. B 66, 052407 (2002).

${ }^{29}$ M. J. Donahue and D. G. Porter, "OOMMF User's Guide, Version 1.0," Interagency Report No. NISTIR 6376, National Institute of Standards and Technology, Gaithersburg, MD, 1999.

${ }^{30}$ S. Chikazumi, Physics of Magnetism (Krieger Publishing Co., Malabar, FL, 1978), p. 186.

${ }^{31}$ L. Thevenard, H. T. Zeng, D. Petit, and R. P. Cowburn, J. Appl. Phys. 106, 063902 (2009).

${ }^{32}$ D. K. Koltsov, R. P. Cowburn, and M. E. Welland, J. Appl. Phys. 88, 5315 (2000).

${ }^{33}$ F. Cebollada, A. Hernando-Mañeru, A. Hernando, C. Martinez-Boubeta, A. Cebollada, and J. M. Gonzalez, Phys. Rev. B 66, 174410 (2002). 